\title{
Ceftobiprole medocaril in the treatment of hospital-acquired pneumonia
}

Thomas WL Scheeren*

Ceftobiprole medocaril is a fifth-generation cephalosporin approved in Europe as singleagent therapy for hospital-acquired pneumonia (HAP), excluding ventilator-associated pneumonia (VAP). It is rapidly converted to the active metabolite ceftobiprole following intravenous administration. Ceftobiprole has a broad spectrum of activity, notably against methicillin-resistant Staphylococcus aureus, ampicillin-susceptible enterococci, penicillinresistant pneumococci and Enterobacteriaceae not producing extended-spectrum $\beta$-lactamase. Ceftobiprole is primarily excreted renally by glomerular filtration, with minimal propensity for interaction with co-administered drugs. Normal dose is ceftobiprole $500 \mathrm{mg}$, administered by 2 -h intravenous infusion every $8 \mathrm{~h}$, with dose adjustment according to renal function. In a pivotal Phase III trial in patients with HAP, ceftobiprole monotherapy was as efficacious as ceftazidime/linezolid for clinical and microbiological cure and was noninferior to ceftazidime/linezolid in the subgroup of patients with HAP excluding VAP. Ceftobiprole and ceftazidime/linezolid were similarly well tolerated. Ceftobiprole is an efficacious and well-tolerated option for empirical treatment of patients with HAP (excluding VAP).

Hospital-acquired pneumonia (HAP) is one of the most common nosocomial infections, with an incidence of 5-10 cases per 1000 hospital admissions in the USA, approximately one-quarter of which are acquired in intensive care units (ICUs) [1-3]. Higher rates of HAP occur in elderly people, patients who are immunocompromised or have undergone surgery, and those being fed via a nasogastric tube [2]. HAP is associated with high mortality and morbidity, and consequently increases the burden on healthcare resources. HAP prolongs hospital stay by 7-9 days on average, and is associated with excess costs of more than US $\$ 40,000$ per patient [2]. The crude death rate among patients with HAP is $30-70 \%$ [2].

The most common bacterial pathogens involved in HAP are Staphylococcus aureus (including methicillin-resistant S. aureus [MRSA]), Pseudomonas aeruginosa, Acinetobacter spp. and some Enterobacteriaceae spp. (such as Klebsiella spp., Enterobacter spp. and Escherichia coli) and less frequently, Serratia spp., Stenotrophomonas maltophila, Streptococcus pneumoniae and Haemophilus influenzae [4].

Initial empirical antimicrobial therapy for HAP needs to target a broad spectrum of Gram-positive and Gram-negative pathogens. The choice of therapeutic agent should take into consideration the types and susceptibility patterns of local pathogens (including those within a particular hospital), as well as other variables such as the duration of previous hospital stay (e.g., for differentiation between early- and late-onset HAP), recent use of antibiotic therapy and the presence of comorbidities [2,5-7]. Appropriate empirical therapy should be implemented rapidly (within $4 \mathrm{~h}$ ) following the initial diagnosis $[7,8]$; guidelines list a range of treatment options including $\beta$-lactams/ $\beta$-lactamase inhibitors

*Department of Anesthesiology, University Medical Center Groningen, University of Groningen, Hanzeplein 1, 9713 GZ, Groningen, The Netherlands; Tel.: +31 50361 6161; Fax: +31 50361 3763; t.w.l.scheeren@umcg.nl

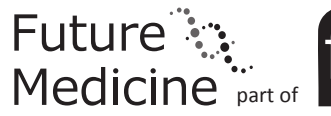

\section{KEYWORDS}

- antimicrobial

- bacteria $\bullet$ ceftobiprole

- cephalosporins • Gramnegative $\bullet$ Gram-positive - hospital-acquired pneumonia 
(e.g., piperacillin/tazobactam), cephalosporins (e.g., cefuroxime, cefotaxamine, ceftriazone and ceftazidime), carbapenems (e.g., imipenem and meropenem) and fluoroquinolones (e.g., levofloxacin, moxifloxacin and ciprofloxacin) $[2,6]$. Suspicion of the involvement of 'hard-to-treat' pathogens, such as $P$. aeruginosa, Acinetobacter spp. or MRSA, further directs the treatment (e.g., suspicion of MRSA requires the use of vancomycin, linezolid or teicoplanin) [6]. There remains a need for additional antibiotics that provide well-tolerated broad-spectrum activity against multidrug-resistant (MDR) pathogens to simplify initial empirical therapy for HAP.

A range of new antibiotics that might have potential in the treatment of HAP include novel glycopeptides (e.g., dalbavancin, oritavancin and telavancin), iclaprim, temocillin and newgeneration cephalosporins (e.g., ceftobiprole and ceftaroline) [3]. Among these, ceftobiprole is approved in Europe for use as a single-agent therapy for HAP (excluding ventilator-associated pneumonia [VAP]). The aim of this review is to provide an overview of the clinical pharmacology and clinical use of ceftobiprole in patients with HAP. Ceftobiprole is also indicated for use in community-acquired pneumonia (CAP; for reviews, see Syed [9] and Liapikou et al. [10]) and has also been investigated in the treatment of complicated skin and soft-tissue infections (cSSTI; for review, see Zhanel et al. [11]). However, these indications are outside the scope of this review and any information relating to these conditions is included only when it provides supporting evidence.

\section{Introduction to ceftobiprole medocaril}

Ceftobiprole medocaril (Zevtera ${ }^{\circledR}$ or Mabelio ${ }^{\circledR}$; Basilea Pharmaceutica Ltd, Basel, Switzerland) is a prodrug that is rapidly and almost completely metabolized to the active drug, ceftobiprole, following intravenous (iv.) administration (Figure 1). The dose of the drug is reported and measured as the active principal (ceftobiprole $500 \mathrm{mg}$ is administered as ceftobiprole medocaril sodium $666.6 \mathrm{mg}$ ). Ceftobiprole is a member of the pyrrolidinone-3-ylidenemethyl cephem series of cephalosporins [12]. It has been described as a fifthgeneration cephalosporin because of its broad spectrum of activity, including against MRSA, ampicillin-susceptible enterococci, penicillinresistant pneumococci and Enterobacteriaceae strains not producing extended-spectrum $\beta$-lactamase (ESBL) [13]. The recommended dose for patients with normal renal function is ceftobiprole $500 \mathrm{mg}$ by iv. infusion over $2 \mathrm{~h}$ every $8 \mathrm{~h} \mathrm{(q8h)}$ in adults; dose adjustment is required in certain patient groups (see later for details).

The antibacterial activity of ceftobiprole results from binding to penicillin-binding proteins (PBPs), blocking their transpeptidase activity and thus disrupting the synthesis of the peptidoglycan layer of bacterial cell walls. Ceftobiprole binds with strong affinity to important PBPs (e.g., PBP2 and PBP3) in Gram-negative bacteria including E. coli and $P$. aeruginosa, but not to PBP5 in E. coli, or to PBP5 or PBP6 in P. aeruginosa [14]. This may be of relevance because PBP5 plays a role in intrinsic resistance to $\beta$-lactam antibiotics in $E$. coli, as well as playing a key role in maintaining cell shape [15]. In $P$. aeruginosa, the $\beta$-lactamase activity of PBP5 contributes to $\beta$-lactam resistance [16,17]. For Gram-positive bacteria, ceftobiprole has high affinity for PBP2a (encoded by $m e c A$ ), which confers methicillin resistance in MRSA isolates [14]. This activity against MRSA is maintained in strains expressing mecC and mecALGA251 [18]. Ceftobiprole has high affinity for PBP1a and PBP2x, but low affinity for PBP $2 b$ (these three PBPs confer $\beta$-lactam resistance in $S$. pneumoniae [14]). In addition, ceftobiprole lacks affinity for mutated PBP5 (overexpression of which confers penicillin resistance in Enterococcus spp.) [12], although it shows good affinity for the unmutated form, which is overexpressed in some penicillin-resistant strains [19]. The range of PBP binding seen with ceftobiprole implies a broad spectrum of antimicrobial activity in a single agent for important pathogens encountered in patients with HAP.

The activity of ceftobiprole against clinically relevant pathogens has been extensively reviewed elsewhere [9,11]. Table 1 summarizes the in vitro antimicrobial activity of ceftobiprole and comparator agents against pathogens frequently associated with HAP [20]. In vitro, ceftobiprole exhibits activity against major Gram-positive pathogens similar to that of linezolid, teicoplanin and vancomycin [9]. Ceftobiprole has potent activity against $S$. aureus, including MRSA and $S$. aureus strains resistant to linezolid or daptomycin, vancomycin-intermediate $S$. aureus (VISA), heterogeneous VISA and vancomycin-resistant S. aureus (VRSA) [21]. Susceptibility to ceftobiprole was generally maintained across $S$. aureus strains with different staphylococcal cassette chromosome mec and/or multilocus sequence 


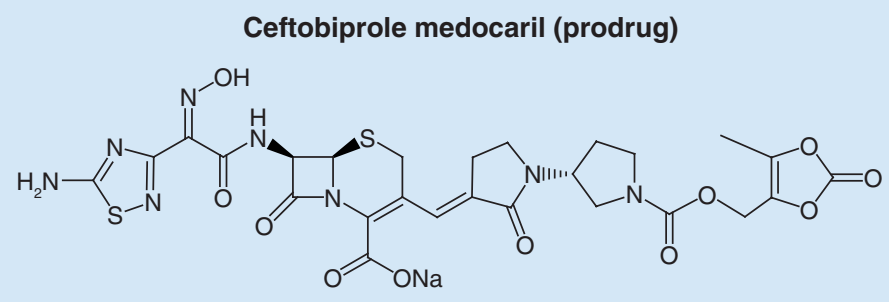

Ceftobiprole (active metabolite)

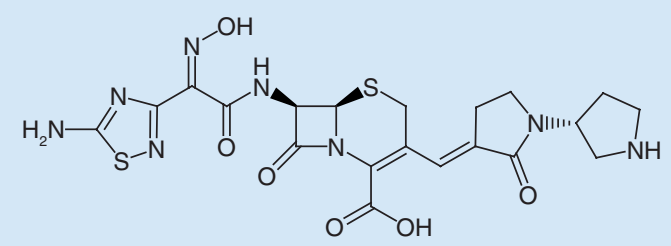

Figure 1. Chemical structures of the prodrug ceftobiprole medocaril and the active metabolite ceftobiprole.

types [21,22]. Ceftobiprole is also active against $S$. pneumoniae, including penicillin-susceptible and penicillin-resistant strains, and $\beta$-hemolytic or viridans streptococci, and Enterococcus faecalis, but not against Enterococcus faecium [9].

With respect to Gram-negative pathogens, ceftobiprole shows good activity against Enterobacteriaceae spp., E. coli, Klebsiella pneumoniae and Proteus mirabilis strains not producing ESBL (Table 1) [20]. However, ceftobiprole was generally inactive against ESBL-producing strains, as were ceftazidime and ceftriaxone [9]. Although Enterobacter spp. and Citrobacter spp. are generally susceptible to ceftobiprole, strains overproducing AmpC $\beta$-lactamase tend to be resistant; ceftazidime and ceftriaxone show more limited activity against these organisms and are also inactive against strains overproducing AmpC $\beta$-lactamase $[9,23]$. In addition, ceftobiprole shows good activity against ceftazidime-susceptible strains of Serratia spp., including Serratia marcescens [9], and approximately two-thirds of $P$. aeruginosa isolates are susceptible to ceftobiprole (Table 1). Ceftobiprole has limited activity against Acinetobacter spp. (Table 1), but exhibits good activity against Gram-negative fastidious respiratory pathogens such as $H$. influenzae and Moraxella catarrhalis irrespective of $\beta$-lactamase production or ampicillin resistance [9].

For anaerobic bacteria, ceftobiprole is generally active against Clostridium spp. and Fusobacterium spp., but inactive against Bacteroides spp., Prevotella spp. and Veillonella spp. [11]. Bacteria causing atypical pneumonia (Chlamydophila pneumoniae, Burkholderia cepacia complex, Mycoplasma pneumoniae, Mycobacterium spp., Norcardia spp. and Stenotrophomonas maltophila) are not generally susceptible to ceftobiprole [18].

Ceftobiprole has demonstrated in vitro bactericidal activity toward all or most MRSA clinical isolates, including heterogeneous VISA, VISA, VRSA and coagulase-negative strains [22,24-28]. Moreover, ceftobiprole is effective in the prevention of intracellular MRSA growth in macrophages and keratinocytes compared with other cephalosporins, such as ceftriaxone, cefoxitin, cephalexin or cefuroxime; the increased activity of ceftobiprole compared with the other cephalosporins was at least partly related to its greater ability to bind to PBP2a under acidic conditions [29]. Ceftobiprole is generally bactericidal in vitro against $S$. pneumoniae (including strains resistant to penicillin, macrolides and/or fluoroquinolones), vancomycin-resistant $E$. faecalis, $H$. influenzae (including $\beta$-lactamase-positive strains), $\beta$-lactamase-positive $M$. catarrhalis and ESBL-negative Enterobacter cloacae [30-33]. Ceftobiprole has also demonstrated in vitro bactericidal activity against $P$. aeruginosa [30,34]. In addition, it exhibits a modest postantibiotic effect against 12 pneumococcal, staphylococcal and enterococcal isolates in vitro [35], and against an MRSA strain, but minimal postantibiotic effects were observed against a penicillin-resistant S. pneumoniae strain in vivo [36]. 
Table 1. In vitro activities of ceftobiprole and comparator agents against key bacterial isolates from European, Turkish and Israeli medical centers (2005-2010).

\begin{tabular}{|c|c|c|c|c|}
\hline \multicolumn{5}{|c|}{ All Staphylococcus aureus $(n=15,426)$} \\
\hline Ceftriaxone & $\leq 0.25->8$ & 4 & $>8$ & 73 \\
\hline Linezolid & $\leq 0.12->8$ & 1 & 2 & $>99$ \\
\hline \multicolumn{5}{|c|}{ Methicillin/oxacillin-susceptible S. aureus $(n=11,279)$} \\
\hline Ceftobiprole & $\leq 0.06-2$ & 0.25 & 0.5 & 100 \\
\hline Ceftriaxone & $\leq 0.25->8$ & 4 & 4 & 100 \\
\hline Linezolid & $\leq 0.12-2$ & 1 & 2 & 100 \\
\hline Vancomycin & $\leq 0.12-2$ & 1 & 1 & 100 \\
\hline \multicolumn{5}{|c|}{ Methicillin/oxacillin-resistant S. aureus $(n=4147)$} \\
\hline \multicolumn{5}{|c|}{ Streptococcus pneumoniae $(n=4443)$} \\
\hline Ceftobiprole & $\leq 0.06-2$ & $\leq 0.06$ & 0.5 & 99 \\
\hline Ceftriaxone & $\leq 0.25-32$ & $\leq 0.25$ & 1 & 84 \\
\hline Linezolid & $\leq 0.12-2$ & 1 & 1 & 100 \\
\hline Vancomycin & $\leq 1-1$ & 1 & 1 & 100 \\
\hline \multicolumn{5}{|c|}{ Enterobacteriaceae spp. $(n=17,480)$} \\
\hline Ceftobiprole & $\leq 0.06->8$ & $\leq 0.06$ & $>8$ & 83 \\
\hline Ceftazidime & $\leq 1->16$ & $\leq 1$ & 16 & 84 \\
\hline \multicolumn{5}{|c|}{ Pseudomonas aeruginosa $(n=3434)$} \\
\hline Ceftobiprole & $\leq 0.06->8$ & 2 & $>8$ & $65^{\dagger}$ \\
\hline \multicolumn{5}{|c|}{$\begin{array}{l}\text { 'EUCAST nonspecies-specific breakpoint. } \\
\text { EUCAST: European Committee on Antimicrobial Susceptibility Testing; MIC: Minimum inhibitory concentration; } \text { MIC }_{50} \text { :MIC required to inhibit } 50 \% \text { of isolates; } \text { MIC }_{90} \text { : MIC required } \\
\text { to inhibit } 90 \% \text { of isolates. } \\
\text { Data taken from [20] @ 2014. Reproduced/amended with permission from the American Society for Microbiology. }\end{array}$} \\
\hline
\end{tabular}

Ceftobiprole has demonstrated synergistic activity in combination with vancomycin against MRSA and glycopeptide-intermediate $S$. aureus both in vitro [37] and in vivo [38], and against VISA in vivo [39]. Ceftobiprole has also shown in vitro synergy in time-kill studies in combination with daptomycin against daptomycin-nonsusceptible MRSA strains [40], and some daptomycin-susceptible and daptomycin-resistant/vancomycinresistant enterococci [41]; ceftobiprole has also shown in vitro synergy in combination with plazomicin against some MRSA strains [42], and in combination with amikacin or levofloxacin against $P$. aeruginosa [34]. Ceftobiprole exhibits bactericidal and curative activity in murine pneumonia models of infection by $S$. aureus including MRSA [43,44], S. pneumoniae including MDR strains [45], H. influenzae [46], E. cloacae [46] and ESBL-negative but not ESBL-positive K. pneumoniae [46].

\section{Pharmacokinetics \& pharmacokinetic/ pharmacodynamic considerations}

The pharmacokinetics of ceftobiprole have been extensively reviewed elsewhere [47]. The principal pharmacokinetic parameters of ceftobiprole in healthy individuals are summarized in Table $2[48,49]$. Following iv. administration, ceftobiprole medocaril is rapidly ( $<1 \mathrm{~min})$ and almost completely converted to active ceftobiprole [47]. 
Peak plasma concentrations $\left(\mathrm{C}_{\max }\right)$ are reached at the end of infusion and show a biphasic decline as a result of initial rapid distribution to other body compartments, principally the extracellular fluid, followed by a relatively slow elimination $[48,49]$. The pharmacokinetics of ceftobiprole are linear after single and multiple doses of 125-1000 mg [48,49]. Steady-state drug concentrations are reached on the first day using a $\mathrm{q} 8 \mathrm{~h}$ regimen [47]. Following administration of the approved dose regimen $(500 \mathrm{mg}$ iv. $\mathrm{q} 8 \mathrm{~h}$ administered over $2 \mathrm{~h}$ ) in healthy individuals, at Day 5 the mean ceftobiprole $\mathrm{C}_{\max }$ was $33 \mathrm{mg} / \mathrm{l}$, the area under the plasma concentration-time curve (AUC) over the dosing interval was 102 $\mathrm{mg} \mathrm{h} / \mathrm{l}$, the volume of distribution at steady state $\left(\mathrm{V}_{\mathrm{ss}}\right)$ was $15.5 \mathrm{l}$ (equating to the volume of the extracellular fluid) [47] and the AUC in epithelial lining fluid versus that for plasma was approximately $25 \%$ [44].

Ceftobiprole has limited potential to interact with other drugs during co-administration. Active ceftobiprole undergoes minimal metabolism to form a microbiologically inactive open-ring metabolite, which accounts for approximately $4 \%$ of ceftobiprole exposure in individuals with normal renal function [47]. Ceftobiprole shows minimal (16\%) binding to plasma protein (primarily albumin but also $\alpha_{1}$-acidic glycoprotein), which is independent of drug and protein concentrations [47]. In vitro, ceftobiprole is not an inhibitor of and/or substrate for the cytochrome P450 system or the P-glycoprotein transporter system. Moreover, ceftobiprole is not an inhibitor of the breast cancer resistance protein, multidrug resistance protein 1 , multidrug resistance-associated protein 2, organic anion transporters (OAT) 1 or OAT 3, or organic cation transporters 1 and 2 [9]. Ceftobiprole is an inhibitor of hepatocyte uptake transporters, organic anion transporting polypeptide 1B1 (OATP1B1) and OATP1B3, and may therefore increase plasma concentrations of drugs that are metabolized by these pathways (e.g., statins, glyburide and bosentan) [9].

Ceftobiprole is almost exclusively eliminated through renal (glomerular filtration) excretion, with a mean elimination half-life $\left(\mathrm{t}_{1 / 2}\right)$ of $3.3 \mathrm{~h}$, a total systemic clearance (CL) of $5.0 \mathrm{l} / \mathrm{h}$ and a total renal clearance $\left(\mathrm{CL}_{\mathrm{R}}\right)$ of $4.3 \mathrm{l} / \mathrm{h}$ [47]. About $88 \%$ of the dose administered is recovered in urine, of which $80-90 \%$ is active ceftobiprole [9]. The risk of renal drug-drug interactions is low given that ceftobiprole does not undergo active tubular excretion and undergoes minimal reabsorption [9].

Ceftobiprole undergoes minimal hepatic metabolism; it is slowly converted to the inactive open-ring metabolite in hepatocytes [47]. Fecal excretion of active ceftobiprole is also minimal, with no measurable concentrations of ceftobiprole detected in feces from healthy volunteers who received iv. ceftobiprole $500 \mathrm{mg}$ q8h for 7 days [50]. The minimal hepatic metabolism and fecal excretion of active ceftobiprole may indicate that the drug is likely to have a negligible impact on intestinal microflora. Indeed, ceftobiprole did not promote $C$. difficile growth and toxin production in murine cecal contents, unlike ceftazidime, cefoxitin, ceftriaxone, cefotaxime and ertapenem, which did undergo at least some degree of hepatic metabolism and fecal excretion [51]. Furthermore, administration of ceftobiprole $500 \mathrm{mg}$ q8h for 1 week had no significant effect on normal intestinal microflora in healthy humans [50]. Taken together, these properties may help minimize the potential for ceftobiprole to be associated with Clostridium difficile infection, which can be driven by broadspectrum antimicrobials undergoing hepatic metabolism and subsequent biliary excretion [52].

Given the predominantly renal elimination, ceftobiprole disposition is affected by renal impairment. In a study of 20 otherwise healthy volunteers, systemic ceftobiprole exposure increased with decreasing renal function [18,53,54]. Ceftobiprole AUC increased by approximately $30 \%, 250 \%$ and $330 \%$ in individuals with mild (creatinine clearance $\left[\mathrm{CL}_{\mathrm{CR}}\right]$ $50-80 \mathrm{ml} / \mathrm{min})$, moderate $\left(\mathrm{CL}_{\mathrm{CR}} 30-<50 \mathrm{ml} /\right.$ min) and severe $\left(\mathrm{CL}_{\mathrm{CR}}<30 \mathrm{ml} / \mathrm{min}\right)$ renal impairment, respectively, compared with those with normal renal function $[18,53,54]$. In addition, patients with moderate or severe renal impairment had reduced renal clearance of ceftobiprole compared with individuals with normal renal function (78 and 91\%, respectively) [9]. The $\mathrm{t}_{1 / 2}$ of ceftobiprole was longest in patients with severe renal impairment (11 vs $3.5 \mathrm{~h}$ in those with normal renal function) [53]. Exposure to ceftobiprole was also shown to be substantially increased in patients with end-stage renal disease (ESRD) [9]. In patients treated in an ICU, pathophysiological changes such as increased cardiac output in sepsis may enhance renal function, increasing $\mathrm{CL}_{\mathrm{CR}}$ to supranormal levels (>150 ml/ min) [55]. Ceftobiprole CL and $\mathrm{V}_{\mathrm{SS}}$ are increased by about 40 and $30 \%$, respectively, in patients 
Table 2. Principal pharmacokinetic parameters for ceftobiprole following single- or multiple-dose administration of intravenous ceftobiprole over $30 \mathrm{~min}$.

\begin{tabular}{|c|c|c|c|c|c|c|c|c|c|}
\hline & \multicolumn{5}{|c|}{ Single dose } & \multicolumn{4}{|c|}{$\begin{array}{l}\text { Multiple doses ( } \mathrm{q} 24 \mathrm{~h} \text { on days } 1 \text { and } 8 ; \mathrm{q} 12 \mathrm{~h} \text { on } \\
\text { days } 2-7 \text { ) }\end{array}$} \\
\hline & $125 \mathrm{mg}$ & $250 \mathrm{mg}$ & $500 \mathrm{mg}$ & $750 \mathrm{mg}$ & $1000 \mathrm{mg}$ & \multicolumn{2}{|c|}{ Day 1} & \multicolumn{2}{|c|}{ Day 8} \\
\hline$C_{\max }(\mathrm{mg} / \mathrm{l})$ & $9.87 \pm 0.78$ & $19.5 \pm 2.75$ & $35.5 \pm 6.79$ & $59.6 \pm 10.7$ & $72.2 \pm 8.78$ & $40.6 \pm 7.38$ & $60.7 \pm 4.55$ & $44.2 \pm 10.8$ & $60.6 \pm 9.99$ \\
\hline $\mathrm{AUC}_{0-\infty}(\mathrm{mg} \mathrm{h} / \mathrm{l})$ & $20.3 \pm 2.82$ & $43.7 \pm 5.99$ & $76.6 \pm 3.88$ & $135 \pm 27.6$ & $151 \pm 9.04$ & $101 \pm 9.04$ & $156 \pm 19.3$ & $108 \pm 22.2$ & $165 \pm 12.8$ \\
\hline $\mathrm{CL}_{\mathrm{R}}(\mathrm{I} / \mathrm{h})$ & $4.60 \pm 0.38$ & $4.35 \pm 0.57$ & $5.07 \pm 0.22$ & $4.08 \pm 0.75$ & $4.16 \pm 0.57$ & $4.12 \pm 0.75$ & $4.05 \pm 0.47$ & $4.47 \pm 1.07$ & $4.09 \pm 0.60$ \\
\hline $\mathrm{V}_{\mathrm{ss}}(\mathrm{I})$ & $17.9 \pm 2.00$ & $17.8 \pm 3.11$ & $19.8 \pm 1.95$ & $18.4 \pm 2.63$ & $18.9 \pm 2.31$ & $16.4 \pm 2.11$ & $16.3 \pm 1.82$ & $16.7 \pm 3.58$ & $16.1 \pm 2.20$ \\
\hline $\begin{array}{l}\text { Urinary recovery } \\
(\%)\end{array}$ & $74.2 \pm 8.14$ & $76.1 \pm 14.4$ & $77.5 \pm 3.27$ & $71.3 \pm 1.48$ & $62.5 \pm 6.33$ & $82.2 \pm 9.11$ & $83.7 \pm 3.91$ & $87.8 \pm 9.30$ & $84.4 \pm 9.23$ \\
\hline \multicolumn{10}{|c|}{ 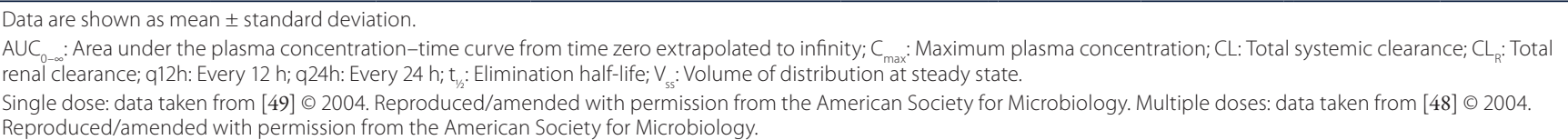 } \\
\hline
\end{tabular}

with augmented renal function compared with values in patients with normal renal function [9]. Similar results have been reported with highdose ceftobiprole $(1000 \mathrm{mg})$ administered by iv. infusion over $4 \mathrm{~h} \mathrm{q} 8 \mathrm{~h}$ in patients in an ICU [56].

Systemic exposure to ceftobiprole is slightly higher (by approximately 20\%) in women compared with men after administration of the same dose, although this is almost exclusively accounted for by difference in body weight [47]. Importantly, the percentage of the dosing interval during which the free drug concentration remained above the minimum inhibitory concentration (MIC; \% $f \mathrm{~T}>\mathrm{MIC}$ ), assuming an MIC of $4 \mathrm{mg} / \mathrm{l}$, is not affected by gender [57]. The pharmacokinetics of ceftobiprole have not yet been determined in adolescents or children ( $<18$ years of age).

The probability of achieving specific percentage targets for $f \mathrm{~T}>\mathrm{MIC}$ with ceftobiprole has been determined using population pharmacokinetic modeling and Monte Carlo simulations for different dose regimens [58,59]. For a MIC cutoff of $4 \mathrm{mg} / \mathrm{l}$ for Gram-positive bacteria, the probability of reaching $40 \%, 50 \%$ and $60 \% f \mathrm{~T}>\mathrm{MIC}$ targets was $100 \%, 99 \%$ and $79 \%$, respectively, for ceftobiprole $500 \mathrm{mg}$ iv. $\mathrm{q} 8 \mathrm{~h}$ administered over $30 \mathrm{~min}$ [58]. In a second set of analyses, ceftobiprole $500 \mathrm{mg}$ iv. q8h administered over $2 \mathrm{~h}$ was associated with an $80 \%$ probability of achieving a $50 \% f \mathrm{~T}>\mathrm{MIC}$ target [59]. The probabilities of achieving a $50 \% f \mathrm{~T}>\mathrm{MIC}$ target were $99 \%$ and $100 \%$ for MRSA ( $\left.\mathrm{MIC}_{90} 1 \mathrm{mg} / \mathrm{l}\right)$ and methicillin-sensitive $S$. aureus $\left(\mathrm{MIC}_{90} 0.5 \mathrm{mg} / \mathrm{l}\right.$ ), respectively, and the probabilities of attaining a
$60 \% f$ T $>$ MIC target were $88 \%, 94 \%$ and $62 \%$ for AmpC-producing bacilli $\left(\mathrm{MIC}_{90} 16 \mathrm{mg} / \mathrm{l}\right)$, AmpC-nonproducing bacilli $\left(\mathrm{MIC}_{90} \leq 0.25 \mathrm{mg} / \mathrm{l}\right)$ and $P$. aeruginosa $\left(\mathrm{MIC}_{90} 32 \mathrm{mg} / \mathrm{l}\right.$ ), respectively. Based on these analyses, the ceftobiprole dosing regimen of $500 \mathrm{mg}$ iv. q8h administered over $2 \mathrm{~h}$ was selected for the Phase III studies in patients with Gram-positive and Gram-negative pathogens. In a validation study [60], the probability of $f \mathrm{~T}>\mathrm{MIC}$ target attainment determined from the Monte Carlo simulations adequately predicted the ceftobiprole plasma concentrations achieved in the Phase III study in patients with HAP.

\section{Clinical efficacy}

The efficacy of ceftobiprole has been investigated in Phase III clinical trials in patients with HAP [61], CAP [62] and cSSTIs [11]. This current review centers on the pivotal Phase III trial in patients with HAP, which compared the efficacy and safety of single-agent therapy with ceftobiprole and the combination of ceftazidime/ linezolid [61].

The pivotal Phase III trial was a multinational, randomized, double-blind, noninferiority study comparing ceftobiprole with ceftazidime/ linezolid in 781 adults with HAP (defined as a diagnosis of pneumonia after $>72 \mathrm{~h}$ of hospitalization or stay in a chronic care unit). Key inclusion criteria were: clinical signs or symptoms of pneumonia (at least two of purulent respiratory secretion, tachypnea or hypoxemia); fever or leukocytosis/leukopenia; new or persistent radiographic infiltrates; and an Acute Physiology and Chronic 
Health Evaluation II (APACHE II) score of 8-25. Key exclusion criteria were: severe renal or hepatic impairment; evidence of infection with ceftobiprole- or ceftazidime-resistant pathogens; clinical conditions likely to interfere with efficacy assessment (e.g., sustained shock, active tuberculosis, lung abscess and postobstructive pneumonia); and systemic antibiotic treatment for $>24 \mathrm{~h}$ in the $48 \mathrm{~h}$ before enrollment. Patients were stratified across treatment arms according to the presence of VAP (defined as a diagnosis of pneumonia developing $>48 \mathrm{~h}$ after mechanical ventilation [yes/no]) and APACHE II score (8-19/20-25); those with VAP were further stratified according to ventilation duration $(</ \geq 5$ days).

Patients were randomly assigned (1:1) to receive ceftobiprole $(500 \mathrm{mg}$ iv. $\mathrm{q} 8 \mathrm{~h}$ administered over $2 \mathrm{~h}[\mathrm{n}=391])$ or ceftazidime plus linezolid ( $2 \mathrm{~g}$ iv. . every $8 \mathrm{~h}$ [q8h] administered over $2 \mathrm{~h}$ plus $600 \mathrm{mg}$ iv. q12h administered over $1 \mathrm{~h}[\mathrm{n}=390])$ for 7-14 days. Patients in the ceftobiprole group also received placebo $\mathrm{q} 12 \mathrm{~h}$ administered by iv. infusion over $1 \mathrm{~h}$ to maintain the double-blind conditions. Additional openlabel treatment with a fluoroquinolone or an aminoglycoside was permitted for patients at risk of pseudomonal infection (the addition of antipseudomonal therapy in each treatment group was prespecified for ethical reasons because this was the first Phase III study of ceftobiprole in which patients infected with pseudomonas were to be recruited). The primary efficacy end point was clinical cure rate at the test of cure (TOC) visit (7-14 days after the last dose of study drug or early termination) in the intent-to-treat (ITT) and clinically evaluable populations, with noninferiority defined using a 15\% margin for 95\% CIs. The. The main secondary end points were microbiological eradication at the TOC visit in the ITT and microbiologically evaluable populations with a valid pathogen at baseline, and 30-day all-cause mortality in the ITT population, as well as safety and tolerability.

The baseline demographic and clinical characteristics of the ITT population were generally similar in the two treatment groups. In the ceftobiprole and ceftazidime/linezolid arms, there were similar proportions of patients aged $>65$ years ( $45 \%$ vs $47 \%)$, and those with APACHE II score $\geq 15$ ( $41 \%$ vs $41 \%$ ), systemic inflammatory response syndrome (72\% vs $73 \%)$, bacteremia ( $10 \%$ vs $12 \%)$, prior antibiotic use within $24 \mathrm{~h}$ of enrollment ( $58 \%$ vs $62 \%$ ) and a valid baseline pathogen $(69 \%$ vs $68 \%)$, although there was a higher proportion of men than women among those receiving ceftobiprole ( $71 \%$ vs $62 \%$ ). Furthermore, the demographics and baseline characteristics of the ITT population and the most frequently isolated baseline pathogens in the microbiologically evaluable population remained similar in both treatment groups for the subgroups of patients with HAP excluding VAP and those with VAP alone. In the overall ITT population, $69 \%$ of patients had a valid pathogen (Gram-positive, 36\%; Gram-negative, 48\%; polymicrobial, 24\%; Pseudomonas spp., $13 \%$; and MRSA, $11 \%)$. In the overall microbiologically evaluable population, the most frequently $(>5.0 \%)$ isolated baseline pathogens were: $S$. aureus $(42 \%)$, including MRSA (17\%); Enterobacteriaceae (38\%), including K. pneumoniae (12\%), E. coli (10\%), Enterobacter spp. (6.3\%) and Proteus spp. (5.1\%); P. aeruginosa (18\%); A. baumannii (9.3\%); S. pneumoniae (7.8\%); and Haemophilus spp. (5.4\%).

Results for the primary efficacy end point, clinical cure at the TOC visit, are presented for the ITT and clinically evaluable populations in Figure 2. Ceftobiprole was noninferior to ceftazidime/ linezolid for patients with HAP (non-VAP and VAP). Additional efficacy end point analyses were performed on subpopulations with HAP (excluding VAP) and VAP according to regulatory guidance [63]. Ceftobiprole was noninferior to ceftazidime/linezolid in patients with HAP (excluding VAP), but not in those with VAP (Figure 2).

For secondary efficacy end points, microbiological eradication rates at the TOC visit in patients with HAP (excluding VAP) were similar in the ceftobiprole and ceftazidime/linezolid groups in the ITT (49\% vs 54\%; difference $-5.0 ; 95 \%$ CI: $-15.3-5.3)$ and microbiologically evaluable (63\% vs $68 \%$; difference -4.6 ; $95 \%$ CI: -16.7-7.6) populations. Clinical cure and microbiological eradication rates by pathogen for patients with HAP (excluding VAP) were similar in both treatment groups (Table 3). Patients with HAP (excluding VAP) in the ITT population also had similar 30 -day all-cause mortality $(16.7 \%$ vs 18.0\%; difference -1.2; 95\% CI: -7.4-5.0) and pneumonia-specific 30 -day mortality $(5.9 \%$ vs $5.6 \%$; difference 0.3 ; 95\% CI: $-3.5-4.1$ ) with ceftobiprole and ceftazidime/linezolid.

Noninferiority of ceftobiprole relative to ceftazidime/linezolid was not demonstrated in the subgroup of patients with VAP (clinical cure rate at the TOC visit: $23 \%$ vs 37\%; Figure 2). Therefore, ceftobiprole is not approved for the 

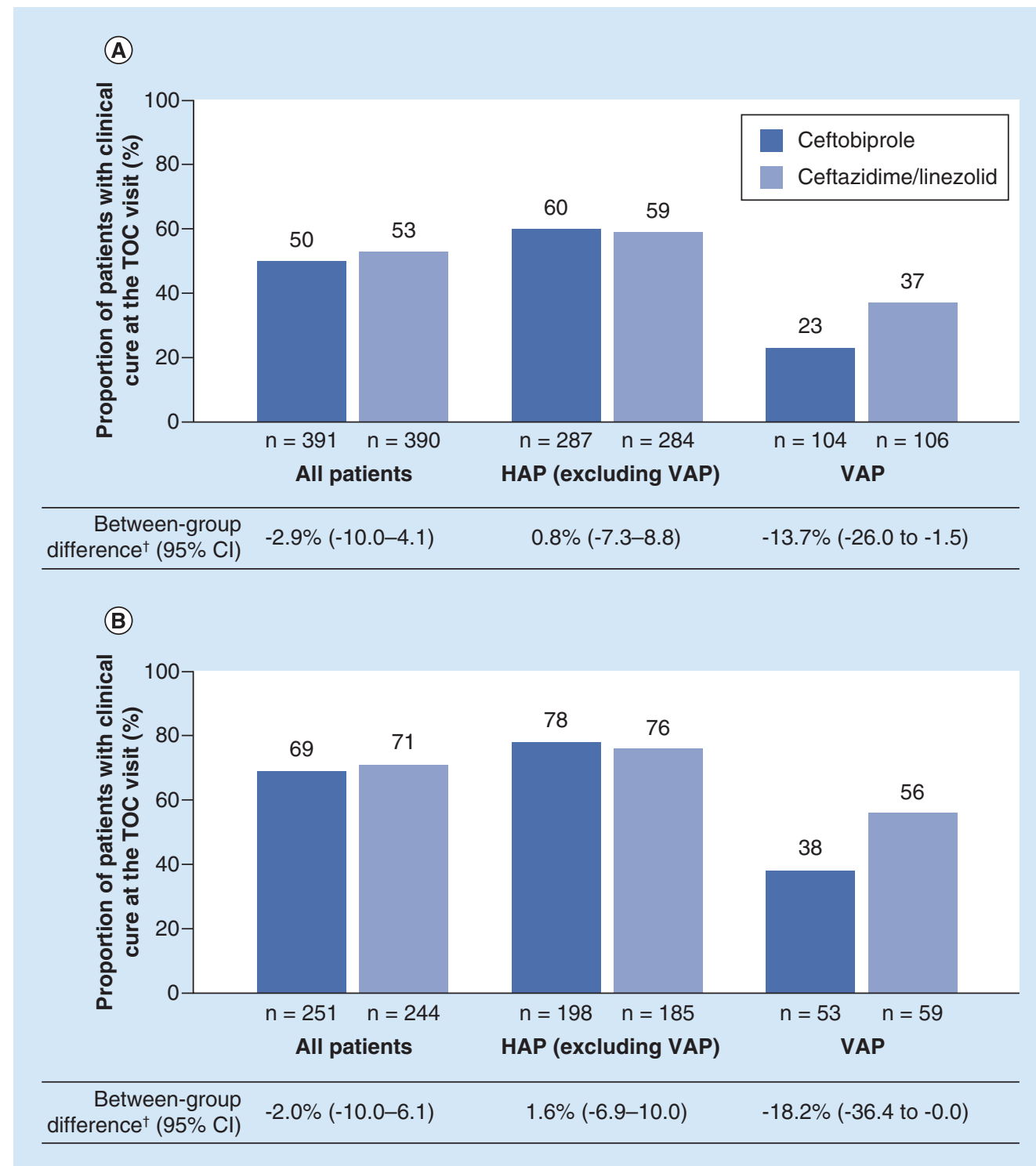

Figure 2. Clinical cure rates with ceftobiprole and ceftazidime/linezolid in the (A) intent-to-treat and (B) clinically evaluable populations.

${ }^{\dagger}$ Difference ceftobiprole minus ceftazidime/linezolid.

HAP: Hospital-acquired pneumonia; TOC: Test of cure; VAP: Ventilator-associated pneumonia.

Data taken from [61].

treatment of patients with VAP. However, analysis of the subgroup of patients who required mechanical ventilation during treatment or developed pneumonia within the $48 \mathrm{~h}$ after the start of ventilation (i.e., who were not defined as having VAP) showed that clinical cure rates were higher with ceftobiprole than with ceftazidime/linezolid ( $55 \%$ vs $41 \%$; difference 14.7\%; 95\% CI: -7.6-37.1). Thus, it appears that mechanical ventilation itself does not explain the results seen in patients with VAP, and ceftobiprole can be used in patients with HAP who require ventilation. Multivariate logistic regression analysis did not identify any of 37 factors (e.g., baseline pathogens, gender, age, comorbidities and vasopressor use) that could explain the outcome in patients with VAP. Moreover, there were no differences between the pharmacokinetic/pharmacodynamic profile of ceftobiprole in the VAP and in the non-VAP subgroups [64]. Modelling data also showed that ceftobiprole plasma concentrations were sufficient for a target MIC 
of $4 \mathrm{mg} / \mathrm{l}$ in $92 \%$ of patients, suggesting that plasma concentrations of ceftobiprole were adequate in patients with VAP [61]. Unfortunately, however, further data exploring the efficacy of ceftobiprole in patients with VAP are not available, and this could be an area for future studies. Consequently, there was no clear explanation for the failure to demonstrate noninferiority in the VAP patients, although a baseline demographic imbalance may have occurred as a result of the relatively small patient numbers enrolled.

Clinical cure rates were further analyzed by baseline demographic and clinical characteristics among patients with HAP (excluding VAP) [61]. Clinical cure rates at the TOC visit in the clinically evaluable population were similar for both treatments in subgroups analyzed by age, gender, patient geographic region, APACHE II score, care facility, prestudy antibiotic use and antipseudomonal antibiotic use (Table 4). The marked difference between treatments in the subgroup of patients in the USA likely reflects the small numbers of patients in each group (ceftobiprole, $\mathrm{n}=27$; ceftazidime/linezolid, $\mathrm{n}=24$ ).

A post hoc analysis of the Phase III trial data was conducted to assess rates of early clinical improvement in patients with HAP (excluding VAP) [65]. Early clinical improvement was defined as improvement of symptoms (including purulent sputum, rales, tachypnea, hypoxemia, pleuritic chest pain, rigor and cough) or cure 4 days after starting treatment, based on an assessment of symptoms by the investigator using standardized criteria. Patients treated with ceftobiprole had a numerically higher early clinical improvement rate than those receiving ceftazidime/linezolid (87\% vs $78 \%$; difference $8.5 \%$; 95\% CI: 0.9-16.1), with more pronounced differences seen in those treated for Gram-positive infections ( $87 \%$ vs $74 \%$; difference $13.0 \%$; 95\% CI: -0.4-26.4), particularly MRSA (95\% vs 53\%; difference $42.1 \%$; 95\% CI: 17.5-66.7). Clinical cure rates at the TOC visit were similar in both treatment groups for the overall population and in the subgroups with Gram-positive or MRSA infections. The higher early clinical improvement rates among patients treated with ceftobiprole than among those receiving ceftazidime/linezolid may be related to the rapid bactericidal profile of ceftobiprole (particularly against Gram-positive organisms) [65].

Ceftobiprole also provides similar efficacy to ceftazidime/linezolid in early- and late-onset HAP (excluding VAP), according to a post hoc analysis of the Phase III trial data [66]. The time of HAP onset is often regarded as an important variable in HAP management, with late-onset disease being more likely to involve MDR pathogens and to be associated with increased morbidity and mortality $[2,67]$. Demographics and clinical

Table 3. Clinical cure rates and microbiological eradication rates with ceftobiprole and ceftriaxone/linezolid at the test of cure visit by pathogen in the microbiologically evaluable population for patients with hospital-acquired pneumonia (excluding ventilator-associated pneumonia).

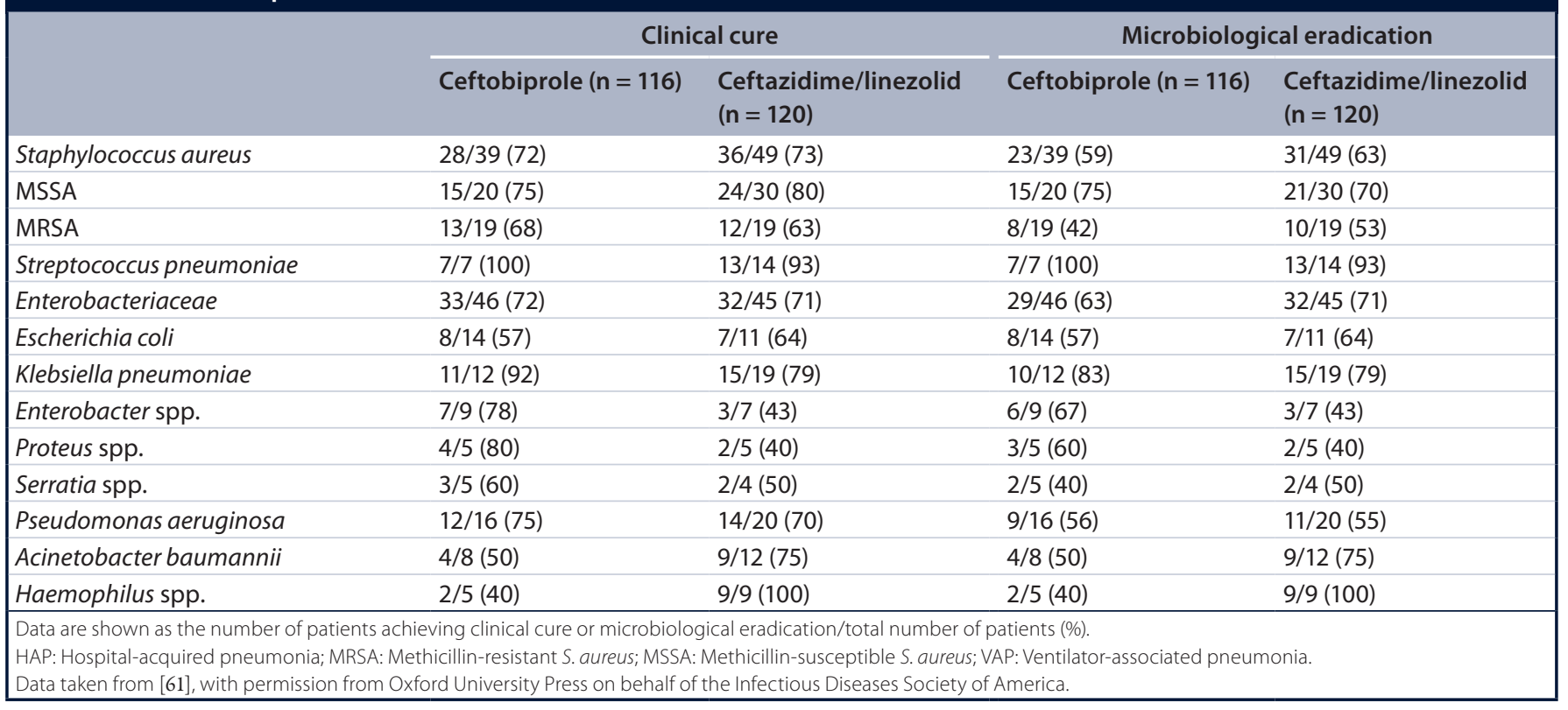


Table 4. Clinical cure rates with ceftobiprole or ceftriaxone/linezolid at the test of cure visit in the clinically evaluable population for patients with hospital-acquired pneumonia (excluding ventilator-associated pneumonia).

\begin{tabular}{|c|c|c|c|}
\hline & Ceftobiprole & Ceftazidime/linezolid & Percent difference $(95 \% \mathrm{Cl})$ \\
\hline All patients & $154 / 198(78)$ & 141/185 (76) & $1.6(-6.9-10.0)$ \\
\hline \multicolumn{4}{|l|}{ Age (years) } \\
\hline $45-64$ & $48 / 60(80)$ & $52 / 66(79)$ & $1.2(-12.9-15.3)$ \\
\hline$\geq 65$ & $82 / 111(74)$ & $71 / 97(73)$ & $0.7(-11.3-12.7)$ \\
\hline \multicolumn{4}{|l|}{ Sex } \\
\hline Female & $47 / 59(80)$ & $55 / 73(75)$ & $4.3(-9.9-18.6)$ \\
\hline \multicolumn{4}{|c|}{ Geographic region } \\
\hline USA & $20 / 27(74)$ & $14 / 24(58)$ & $15.7(-10.0-41.5)$ \\
\hline Europe & $93 / 112(83)$ & $90 / 104(87)$ & $-3.5(-13.1-6.1)$ \\
\hline Other & $41 / 59(69)$ & $37 / 57(65)$ & $4.6(-12.5-21.7)$ \\
\hline \multicolumn{4}{|l|}{ Care facility } \\
\hline ICU & $51 / 73(70)$ & $39 / 59(66)$ & $3.8(-12.3-19.8)$ \\
\hline Non-ICU & $103 / 125(82)$ & $102 / 126(81)$ & $1.4(-8.1-11.0)$ \\
\hline \multicolumn{4}{|c|}{ Pre-study antibiotics ${ }^{\dagger}$} \\
\hline None & $44 / 53(83)$ & $49 / 59(83)$ & $0.0(-14.0-13.9)$ \\
\hline Usage $\leq 24 \mathrm{~h}$ & $52 / 65(80)$ & $45 / 59(76)$ & $3.7(-10.8-18.3)$ \\
\hline Usage $>24 \mathrm{~h}$ & $58 / 80(73)$ & $47 / 67(70)$ & $2.4(-12.3-17.0)$ \\
\hline \multicolumn{4}{|c|}{ Antipseudomonal antibiotics ${ }^{\ddagger}$} \\
\hline Yes & $15 / 27(56)$ & $10 / 19(53)$ & $2.9(-26.3-32.2)$ \\
\hline No & 139/171 (81) & $131 / 166(79)$ & $2.4(-6.2-10.9)$ \\
\hline
\end{tabular}

characteristics as well as baseline pathogens were generally similar in patients with early-onset HAP and those with late-onset disease $(<5$ and $\geq 5$ days after hospitalization), although patients with early-onset HAP tended to be older and were more likely to have received ventilation at baseline. Ceftobiprole was as effective as ceftazidime/linezolid as assessed by clinical cure rates at the TOC visit in both early-onset HAP (75\% vs 67\%; difference $7.7 \%$; 95\% CI: -8.6-24.0) and late-onset HAP (79\% vs $80 \%$; difference $-0.9 \%$; $95 \% \mathrm{CI}$ : -10.6-8.8), for the clinically evaluable population. The 30-day all-cause mortality was also similar between treatments in early- (16\% vs 18\%) and late-onset (17\% vs 18\%) HAP. Interestingly, the similarity of the baseline pathogen profiles in the early- and late-onset HAP subgroups indicates that time to HAP onset is not a strong predictor of pathogen distribution, and this is consistent with the findings of previous studies $[67,68]$.

\section{Safety \& tolerability}

Ceftobiprole is generally well tolerated in patients with HAP. Treatment-related adverse events (AEs) reported in the Phase III study of ceftobiprole in patients with HAP are summarized in Table 5. The AE profile for ceftobiprole was broadly similar to that for ceftazidime/linezolid. The incidences of hyponatremia, phlebitis, vomiting and hypokalemia were higher with ceftobiprole, while those of diarrhea, rash and elevations in levels of aspartate aminotransferase and alanine aminotransferase were higher with ceftazidime/linezolid; dysgeusia was reported only for patients receiving ceftobiprole. Treatment-related serious AEs occurred at a similar rate with ceftobiprole and ceftazidime/linezolid (3.9 vs 3.1\%). There were no clinically relevant between-group differences in other laboratory values, vital signs, results of physical examinations or electrocardiograms. These findings are similar to those 


\section{EXECUTIVE SUMMARY}

\section{Mechanism of action}

- Ceftobiprole binds to penicillin-binding proteins (PBPs), blocking their transpeptidase activity and thus disrupting the synthesis of the peptidoglycan layer of bacterial cell walls.

- Ceftobiprole binds with strong affinity to important PBPs (e.g., PBP2 and PBP3) in Gram-negative bacteria including Escherichia coli and Pseudomonas aeruginosa, but not to PBP5 in E. coli, or to PBP5 or PBP6 in P. aeruginosa.

- For Gram-positive bacteria, ceftobiprole has high affinity for PBP2a (encoded by mecA), which confers methicillin resistance in methicillin-resistant Staphylococcus aureus (MRSA) isolates.

- Ceftobiprole has high affinity for PBP1a and PBP2x, but low affinity for PBP2b (these three PBPs confer $\beta$-lactam resistance in Streptococcus pneumoniae) and mutated PBP5 (which confers penicillin-resistance in Enterococcus spp.).

\section{Microbiology \& spectrum of activity}

- Ceftobiprole exhibits antimicrobial activity in vitro against major Gram-positive pathogens, similar to that of linezolid, teicoplanin and vancomycin.

- Ceftobiprole has potent activity against S. aureus, including MRSA; notably, it maintains activity against S. aureus strains resistant to linezolid or daptomycin, vancomycin-intermediate S. aureus, heterogeneous vancomycin-intermediate $S$. aureus and vancomycin-resistant $S$. aureus.

- With respect to Gram-negative pathogens, a high proportion of Enterobacteriaceae clinical isolates are susceptible to ceftobiprole. Ceftobiprole shows good activity against E. coli, Klebsiella pneumoniae and Proteus mirabilis strains not producing extended-spectrum $\beta$-lactamase (ESBL). However, it is generally inactive against ESBL-producing strains (as are other cephalosporins).

- Citrobacter spp. and Enterobacter spp. are generally susceptible to ceftobiprole; however, strains overproducing AmpC $\beta$-lactamase tend to be resistant.

- For anaerobic bacteria, ceftobiprole is generally active against Clostridium spp. and Fusobacterium spp., but inactive against Bacteroides spp., Prevotella spp. and Veillonella spp.; bacteria causing atypical pneumonia are not generally susceptible to ceftobiprole.

\section{Pharmacokinetic properties}

- Ceftobiprole medocaril is administered by intravenous infusion, and is rapidly ( $<1 \mathrm{~min}$ ) and almost completely converted to active ceftobiprole.

- The pharmacokinetics of ceftobiprole are linear after single and multiple doses over the 125-1000 mg dose range.

- Ceftobiprole undergoes minimal metabolism to form an inactive metabolite and shows minimal (16\%) binding to plasma protein.

- Ceftobiprole is almost exclusively eliminated through renal (glomerular filtration) excretion, with about $88 \%$ of the administered dose recovered in urine; dose adjustments may be required depending on renal function.

- The results of in vitro inhibitor/substrate studies indicate a minimal propensity for interaction with co-administered drugs.

\section{Clinical efficacy}

- A pivotal Phase III trial has demonstrated the efficacy of single-agent therapy with ceftobiprole $500 \mathrm{mg}$ iv. every $8 \mathrm{~h}$ (q8h) administered over $2 \mathrm{~h}$ in patients with hospital-acquired pneumonia (HAP) compared with a combination of ceftazidime/linezolid.

- Ceftobiprole showed noninferiority to ceftazidime/linezolid for clinical cure rates at the test of cure visit in the intentto-treat (50 vs 53\%, respectively) and clinically evaluable (69 vs 71\%, respectively) populations.

- Ceftobiprole was noninferior to ceftazidime/linezolid for clinical cure rates in the subgroup of patients with HAP (excluding ventilator-associated pneumonia [VAP]), but not in those with VAP.

- Clinical cure and microbiological eradication rates by pathogen for patients with HAP (excluding VAP) were similar in both treatment groups. 


\section{EXECUTIVE SUMMARY (CONT.)}

\section{Safety \& tolerability}

- Ceftobiprole is generally well tolerated in patients with HAP.

- The adverse event profile for ceftobiprole was broadly similar to that for ceftazidime/linezolid in the Phase III study in patients with HAP.

- The most common adverse events with ceftobiprole in clinical trials (occurring in $\geq 3 \%$ of patients) were nausea, vomiting, diarrhea, infusion site reactions, hypersensitivity and dysgeusia.

\section{Dosage \& administration}

- The recommended dose regimen for patients with normal renal function is ceftobiprole $500 \mathrm{mg}$ iv. q8h, administered over $2 \mathrm{~h}$ in adults.

- Dose adjustment may be necessary depending on renal function; doses need to be reduced in patients with moderate or severe renal impairment and in those with end-stage renal disease.

- No dose adjustment is needed based on gender, age, body weight or hepatic impairment.

inhibitor/substrate studies indicate minimal propensity for interaction with co-administered drugs. Early-phase clinical studies have established a ceftobiprole regimen of $500 \mathrm{mg}$ iv. q8h administered over $2 \mathrm{~h}$. Dose adjustment may be necessary depending on renal function.

A range of new antibiotics is being investigated that might have potential in the treatment of HAP, including novel glycopeptides (e.g., dalbavancin, oritavancin and telavancin), iclaprim, temocillin and new-generation cephalosporins (e.g., ceftaroline) [3]. Among these agents, ceftaroline also has anti-MRSA activity [70]. Ceftaroline has been approved for the treatment of CAP, but not HAP. There are currently no registered clinical trials of ceftaroline underway, although there has been a report on clinical experience with ceftaroline in a limited number of patients with HAP or VAP [71]. The availability of additional antibiotics that provide well-tolerated broad-spectrum activity against MDR pathogens will help to simplify initial empirical therapy for HAP.

In a pivotal Phase III trial in patients with HAP, empirical therapy with single-agent ceftobiprole showed similar efficacy to the ceftazidime/linezolid combination with respect to clinical and microbiological cure rates. Ceftobiprole was also noninferior to ceftazidime/linezolid in the subgroup of patients with HAP excluding VAP, although noninferiority was not demonstrated in individuals with VAP. Thus, ceftobiprole has been approved in the EU for the treatment of HAP (excluding VAP) and can be used in patients with HAP who require ventilation. It is also approved in the EU for the treatment of CAP. Ceftobiprole and ceftazidime/linezolid were similarly well tolerated in the Phase III trial in patients with HAP. The most common treatment-related AEs across all clinical studies included nausea, vomiting, diarrhea, infusion site reactions, hypersensitivity and dysgeusia.

In conclusion, ceftobiprole is an effective and well-tolerated option for the empirical treatment of patients with HAP (excluding VAP). Its broad spectrum of activity against bacterial pathogens commonly encountered in patients with HAP, including MRSA, offers the potential for a simplified monotherapy regimen compared with the usual combination therapy. It would be important for future studies to explore in more detail the activity of ceftobiprole against hard-to-treat HAP pathogens; in particular, a study assessing the efficacy of ceftobiprole compared with vancomycin or linezolid in MRSA HAP would help further characterize the role of ceftobiprole.

\section{Disclaimer}

The author takes full responsibility for the content of the article.

\section{Financial \& competing interests disclosure}

$T W L$ Scheeren received honoraria for lectures from Basilea

Pharmaceutica, the producer of ceftobiprole. The author

has no other relevant affiliations or financial involvement with any organization or entity with a financial interest in or financial conflict with the subject matter or materials discussed in the manuscript apart from those disclosed.

The author thanks Oxford PharmaGenesis Ltd (Oxford, $U K)$, who provided medical writing and editorial support; this was funded by Basilea Pharmaceutica International Ltd (Basel, Switzerland). 


\section{References}

Papers of special note have been highlighted as: - of interest; $\bullet \bullet$ of considerable interest

1 Richards MJ, Edwards JR, Culver DH, Gaynes RP. Nosocomial infections in medical intensive care units in the United States. National Nosocomial Infections Surveillance System. Crit. Care Med. 27(5), 887-892 (1999).

2 American Thoracic Society-Infectious Diseases Society of America. Guidelines for the management of adults with hospitalacquired, ventilator-associated, and healthcare-associated pneumonia. Am. J. Respir. Crit. Care Med. 171(4), 388-416 (2005).

3 Barbier F, Andremont A, Wolff M, Bouadma L. Hospital-acquired pneumonia and ventilator-associated pneumonia: recent advances in epidemiology and management. Curr. Opin. Pulm. Med. 19(3), 216-228 (2013).

4 Jones RN. Microbial etiologies of hospitalacquired bacterial pneumonia and ventilatorassociated bacterial pneumonia. Clin. Infect. Dis. 51(Suppl. 1),S81-S87 (2010).

5 Masterton RG, Galloway A, French G et al. Guidelines for the management of hospitalacquired pneumonia in the UK: report of the working party on hospital-acquired pneumonia of the British Society for Antimicrobial Chemotherapy. J. Antimicrob. Chemother. 62(1), 5-34 (2008).

6 Torres A, Ferrer M, Badia JR. Treatment guidelines and outcomes of hospitalacquired and ventilator-associated pneumonia. Clin. Infect. Dis. 51(Suppl. 1), S48-S53 (2010).

7 Eccles S, Pincus C, Higgins B, Woodhead M. Diagnosis and management of community and hospital acquired pneumonia in adults: summary of NICE guidance. BMJ 349, g6722 (2014).

8 Kollef M. Appropriate empirical antibacterial therapy for nosocomial infections: getting it right the first time. Drugs 63(20), 2157-2168 (2003).

9 Syed YY. Ceftobiprole medocaril: a review of its use in patients with hospital- or community-acquired pneumonia. Drugs 74(13), 1523-1542 (2014).

- Presents a review of ceftobiprole in hospital-acquired pneumonia (HAP) and community-acquired pneumonia (CAP).

10 Liapikou A, Cilloniz C, Torres A. Ceftobiprole for the treatment of pneumonia: a European perspective. Drug Des. Devel. Ther. 9, 4565-4572 (2015).
11 Zhanel GG, Lam A, Schweizer F et al. Ceftobiprole: a review of a broad-spectrum and anti-MRSA cephalosporin. Am. J. Clin. Dermatol. 9(4), 245-254 (2008).

12 Hebeisen P, Heinze-Krauss I, Angehrn P, Hohl P, Page MG, Then RL. In vitro and in vivo properties of Ro 63-9141, a novel broad-spectrum cephalosporin with activity against methicillin-resistant staphylococci. Antimicrob. Agents Chemother. 45(3), 825-836 (2001).

13 Widmer AF. Ceftobiprole: a new option for treatment of skin and soft-tissue infections due to methicillin-resistant Staphylococcus aureus. Clin. Infect. Dis. 46(5), 656-658 (2008).

14 Davies TA, Page MG, Shang W, Andrew T, Kania M, Bush K. Binding of ceftobiprole and comparators to the penicillin-binding proteins of Escherichia coli, Pseudomonas aeruginosa, Staphylococcus aureus and Streptococcus pneumoniae. Antimicrob. Agents Chemother. 51(7), 2621-2624 (2007).

15 Sarkar SK, Dutta M, Chowdhury C, Kumar A, Ghosh AS. PBP5, PBP6 and DacD play different roles in intrinsic beta-lactam resistance of Escherichia coli. Microbiology 157(Pt 9), 2702-2707 (2011).

16 Smith JD, Kumarasiri M, Zhang W et al. Structural analysis of the role of Pseudomonas aeruginosa penicillin-binding protein 5 in beta-lactam resistance. Antimicrob. Agents Chemother. 57(7), 3137-3146 (2013).

17 Ropy A, Cabot G, Sanchez-Diener I et al. Role of Pseudomonas aeruginosa lowmolecular-mass penicillin-binding proteins in AmpC expression, beta-lactam resistance, and peptidoglycan structure. Antimicrob. Agents Chemother. 59(7), 3925-3934 (2015).

18 Basilea Pharmaceutica International Ltd. Summary of Product Characteristics Zevtera. 20 Nov 2013.

http://www.mhra.gov.uk

19 Henry X, Amoroso A, Coyette J, Joris B. Interaction of ceftobiprole with the low-affinity PBP 5 of Enterococcus faecium. Antimicrob. Agents Chemother. 54(2), 953-955 (2010).

20 Farrell DJ, Flamm RK, Sader HS, Jones RN. Ceftobiprole activity against over 60,000 clinical bacterial pathogens isolated in Europe, Turkey, and Israel from 2005 to 2010. Antimicrob. Agents Chemother. 58(7), 3882-3888 (2014).

- Evaluates the in vitro antimicrobial activity of ceftobiprole against prevalent Grampositive and -negative pathogens isolated in Europe from 2005 to 2010.
21 Farrell DJ, Flamm RK, Sader HS, Jones RN. Activity of ceftobiprole against methicillinresistant Staphylococcus aureus strains with reduced susceptibility to daptomycin, linezolid or vancomycin, and strains with defined SCCmec types. Int. J. Antimicrob. Agents 43(4), 323-327 (2014).

- Evaluates the activity of ceftobiprole against MRSA isolates with decreased susceptibility to daptomycin, linezolid or vancomycin.

22 Borbone S, Campanile F, Bongiorno D, Stefani S. In vitro bactericidal activity of ceftobiprole against hospital- and communityassociated methicillin-resistant Staphylococcus aureus. J. Antimicrob. Chemother. 65(3), 591-594 (2010).

23 Pillar CM, Aranza MK, Shah D, Sahm DF. In vitro activity profile of ceftobiprole, an anti-MRSA cephalosporin, against recent gram-positive and gram-negative isolates of European origin. J. Antimicrob. Chemother. 61(3), 595-602 (2008).

24 Deshpande L, Rhomberg PR, Fritsche TR, Sader HS, Jones RN. Bactericidal activity of BAL9141, a novel parenteral cephalosporin against contemporary Gram-positive and Gram-negative isolates. Diagn. Microbiol. Infect. Dis. 50(1), 73-75 (2004).

25 Bogdanovich T, Ednie LM, Shapiro S, Appelbaum PC. Antistaphylococcal activity of ceftobiprole, a new broad-spectrum cephalosporin. Antimicrob. Agents Chemother. 49(10), 4210-4219 (2005).

26 Leonard SN, Cheung CM, Rybak MJ. Activities of ceftobiprole, linezolid, vancomycin, and daptomycin against community-associated and hospital-associated methicillin-resistant Staphylococcus aureus. Antimicrob. Agents Chemother. 52(8), 2974-2976 (2008).

27 Lin G, Appelbaum PC. Activity of ceftobiprole compared with those of other agents against Staphylococcus aureus strains with different resistotypes by time-kill analysis. Diagn. Microbiol. Infect. Dis. 60(2), 233-235 (2008).

28 Zhanel GG, Voth D, Nichol K, Karlowsky JA, Noreddin AM, Hoban DJ. Pharmacodynamic activity of ceftobiprole compared with vancomycin versus methicillin-resistant Staphylococcus aureus (MRSA), vancomycin-intermediate Staphylococcus aureus (VISA) and vancomycin-resistant Staphylococcus aureus (VRSA) using an in vitro model. J. Antimicrob. Chemother. 64(2), 364-369 (2009).

29 Lemaire S, Glupczynski Y, Duval V, Joris B, Tulkens PM, Van Bambeke F. Activities of 
ceftobiprole and other cephalosporins against extracellular and intracellular (THP-1 macrophages and keratinocytes) forms of methicillin-susceptible and methicillinresistant Staphylococcus aureus. Antimicrob. Agents Chemother. 53(6), 2289-2297 (2009).

30 Issa NC, Rouse MS, Piper KE, Wilson WR, Steckelberg JM, Patel R. In vitro activity of BAL9141 against clinical isolates of Gram-negative bacteria. Diagn. Microbiol. Infect. Dis. 48(1), 73-75 (2004).

31 Kosowska K, Hoellman DB, Lin G et al. Antipneumococcal activity of ceftobiprole, a novel broad-spectrum cephalosporin. Antimicrob. Agents Chemother. 49(5), 1932-1942 (2005).

32 Bogdanovich T, Clark C, Ednie L et al. Activities of ceftobiprole, a novel broadspectrum cephalosporin, against Haemophilus influenzae and Moraxella catarrhalis. Antimicrob. Agents Chemother. 50(6), 2050-2057 (2006).

33 Arias CA, Singh KV, Panesso D, Murray BE. Time-kill and synergism studies of ceftobiprole against Enterococcus faecalis including beta-lactamase-producing and vancomycin-resistant isolates. Antimicrob. Agents Chemother. 51(6), 2043-2047 (2007).

34 Kresken M, Korber-Irrgang B, Lauffer J, Decker-Burgard S, Davies T. In vitro activities of ceftobiprole combined with amikacin or levofloxacin against Pseudomonas aeruginosa: evidence of a synergistic effect using time-kill methodology. Int. J. Antimicrob. Agents 38(1), 70-75 (2011).

35 Pankuch GA, Appelbaum PC. Postantibiotic effect of ceftobiprole against 12 Grampositive organisms. Antimicrob. Agents Chemother. 50(11), 3956-3958 (2006).

36 Craig WA, Andes DR. In vivo pharmacodynamics of ceftobiprole against multiple bacterial pathogens in murine thigh and lung infection models. Antimicrob. Agents Chemother. 52(10), 3492-3496 (2008).

37 Entenza JM, Vouillamoz J, Bizzini A, Giddey M, Bille J, Moreillon P. In vitro synergism between ceftobiprole and vancomycin against methicillin-resistant and glycopeptideintermediate Staphylococcus aureus [abstract no. O39]. Clin. Microbiol. Infect. 16(Suppl. 2), S9 (2010).

38 Fernandez J, Abbanat D, Shang W et al. Synergistic activity of ceftobiprole and vancomycin in a rat model of infective endocarditis caused by methicillin-resistant and glycopeptide-intermediate Staphylococcus aureus. Antimicrob. Agents Chemother. 56(3), 1476-1484 (2012).
39 Entenza JM, Veloso TR, Vouillamoz J, Giddey M, Majcherczyk P, Moreillon P. In vivo synergism of ceftobiprole and vancomycin against experimental endocarditis due to vancomycin-intermediate Staphylococcus aureus. Antimicrob. Agents Chemother. 55(9), 3977-3984 (2011).

40 Barber KE, Werth BJ, Ireland CE et al. Potent synergy of ceftobiprole plus daptomycin against multiple strains of Staphylococcus aureus with various resistance phenotypes. J. Antimicrob. Chemother. 69(11), 3006-3010 (2014).

41 Werth BJ, Barber KE, Tran KN et al. Ceftobiprole and ampicillin increase daptomycin susceptibility of daptomycinsusceptible and -resistant VRE. J. Antimicrob. Chemother. 70 (2), 489-493 (2015).

42 Lin G, Ednie LM, Appelbaum PC. Antistaphylococcal activity of ACHN-490 tested alone and in combination with other agents by time-kill assay. Antimicrob. Agents Chemother. 54(5), 2258-2261 (2010).

43 Laohavaleeson S, Tessier PR, Nicolau DP. Pharmacodynamic characterization of ceftobiprole in experimental pneumonia caused by phenotypically diverse Staphylococcus aureus strains. Antimicrob. Agents Chemother. 52(7), 2389-2394 (2008).

44 Rodvold KA, Nicolau DP, Lodise TP et al. Identifying exposure targets for treatment of staphylococcal pneumonia with ceftobiprole. Antimicrob. Agents Chemother. 53(8), 3294-3301 (2009).

45 Azoulay-Dupuis E, Bedos JP, Mohler J, Schmitt-Hoffmann A, Schleimer M, Shapiro S. Efficacy of BAL5788, a prodrug of cephalosporin BAL9141, in a mouse model of acute pneumococcal pneumonia. Antimicrob. Agents Chemother. 48(4), 1105-1111 (2004).

46 Rouse MS, Hein MM, Anguita-Alonso P, Steckelberg JM, Patel R. Ceftobiprole medocaril (BAL5788) treatment of experimental Haemophilus influenza Enterobacter cloacae and Klebsiella pneumoniae murine pneumonia. Diagn. Microbiol. Infect. Dis. 55(4), 333-336 (2006).

47 Murthy B, Schmitt-Hoffmann A. Pharmacokinetics and pharmacodynamics of ceftobiprole, an anti-MRSA cephalosporin with broad-spectrum activity. Clin. Pharmacokinet. 47(1), 21-33 (2008).

- Provides a comprehensive review of the pharmacokinetics and pharmacodynamics of ceftobiprole.

48 Schmitt-Hoffmann A, Nyman L, Roos B et al. Multiple-dose pharmacokinetics and safety of a novel broad-spectrum cephalosporin (BAL5788) in healthy volunteers. Antimicrob. Agents Chemother. 48(7), 2576-2580 (2004).

49 Schmitt-Hoffmann A, Roos B, Schleimer M et al. Single-dose pharmacokinetics and safety of a novel broad-spectrum cephalosporin (BAL5788) in healthy volunteers. Antimicrob. Agents Chemother. 48(7), 2570-2575 (2004).

50 Backstrom T, Panagiotidis G, Beck O et al. Effect of ceftobiprole on the normal human intestinal microflora. Int. J. Antimicrob. Agents 36(6), 537-541 (2010).

51 Nerandzic MM, Donskey CJ. Effect of ceftobiprole treatment on growth of and toxin production by Clostridium difficile in cecal contents of mice. Antimicrob. Agents Chemother. 55(5), 2174-2177 (2011).

52 Lim WS, Baudouin SV, George RC et al. BTS guidelines for the management of community acquired pneumonia in adults: update 2009. Thorax 64(Suppl. 3), iii1-iii55 (2009).

53 Roos B, Schmitt-Hoffmann A, Schleimer M et al. Safety and pharmacokinetics of BAL5788 in healthy subjects with normal or impaired renal function [abstract A-23]. Presented at: 43rd Annual Interscience Conference on Antimicrobial Agents and Chemotherapy. Chicago, IL, USA, 14-17 September 2003.

54 Kisgen J, Whitney D. Ceftobiprole, a broad-spectrum cephalosporin with activity against methicillin-resistant Staphylococcus aureus (MRSA). P T 33(11), 631-641 (2008).

55 Roberts JA, Lipman J. Pharmacokinetic issues for antibiotics in the critically ill patient. Crit. Care Med. 37(3), 840-851 (2009).

56 Torres A, Sanchez-Garcia M, Demeyer I et al. Pharmacokinetics, safety and tolerability of high-dose (1000 mg) ceftobiprole medocaril administered as prolonged infusion in intensive care unit (ICU) patients [abstract \#ECCMD-0277]. Presented at: 25 th European Congress of Clinical Microbiology and Infectious Diseases. Copenhagen, Denmark, 25-28 April 2015.

57 Schmitt-Hoffmann AH, Roos B, Heep M et al. Influence of gender on the pharmacokinetics of BAL-9141 after intravenous infusion of pro-drug BAL-5788. Presented at: 14th European Congress of Clinical Microbiology and Infectious Diseases. Prague, Czech Republic, 1-4 May 2004.

58 Mouton JW, Schmitt-Hoffmann A, Shapiro S, Nashed N, Punt NC. Use of Monte Carlo simulations to select therapeutic doses and provisional breakpoints of BAL9141. Antimicrob. Agents Chemother. 48(5), 1713-1718 (2004). 
59 Lodise TP Jr, Pypstra R, Kahn JB et al. Probability of target attainment for ceftobiprole as derived from a population pharmacokinetic analysis of 150 subjects. Antimicrob. Agents Chemother. 51(7), 2378-2387 (2007).

60 Muller AE, Schmitt-Hoffmann AH, Punt N, Mouton JW. Monte Carlo simulations based on Phase 1 studies predict target attainment of ceftobiprole in nosocomial pneumonia patients: a validation study. Antimicrob Agents Chemother. 57(5), 2047-2053 (2013).

61 Awad SS, Rodriguez AH, Chuang YC et al. A Phase 3 randomized double-blind comparison of ceftobiprole medocaril versus ceftazidime plus linezolid for the treatment of hospital-acquired pneumonia. Clin. Infect. Dis. 59(1), 51-61 (2014).

- Presents the results of the Phase III trial in HAP comparing ceftobiprole with ceftazidime/linezolid.

62 Nicholson SC, Welte T, File TM Jr et al. A randomised, double-blind trial comparing ceftobiprole medocaril with ceftriaxone with or without linezolid for the treatment of patients with community-acquired pneumonia requiring hospitalisation. Int. J. Antimicrob. Agents 39(3), 240-246 (2012).

- Presents the results of the Phase III trial in CAP comparing ceftobiprole with ceftazidime \pm linezolid.
63 Committee for Medicinal Products for Human Use. Addendum to the note for guidance on evaluation of medicinal products indicated for treatment of bacterial infections (CPMP/ EWP/558/95 REV 2) to address indicationspecific clinical data. European Medicines Agency EMA/CHMP/776609/2011 (2012). http://www.ema.europa.eu

64 Muller AE, Punt N, Mouton JW. Exposure to ceftobiprole is associated with microbiological eradication and clinical cure in patients with nosocomial pneumonia. Antimicrob. Agents Chemother. 58(5), 2512-2519 (2014)

65 Scheeren T, Rodriguez A, Zhou X, Saulay M, Engelhardt M. Early clinical improvement and clinical cure in a randomised controlled Phase 3 study of ceftobiprole versus ceftazidime/linezolid in patients with hospital-acquired pneumonia [abstract \#O151]. Presented at: 24th European Congress of Clinical Microbiology and Infectious Diseases. Barcelona, Spain, 10-13 May 2014.

66 Scheeren TWL, Welte T, Capellier G, Sauley $\mathrm{M}$, Engelhardt M. Clinical cure and mortality outcomes with ceftobiprole medocaril versus ceftazidime plus linezolid in patients with early- versus late-onset hospital-acquired pneumonia [abstract \#ECCMD-0284]. Presented at: 25 th European Congress of Clinical Microbiology and Infectious Diseases. Copenhagen, Denmark, 25-28 April 2015.

67 Martin-Loeches I, Deja M, Koulenti D et al. Potentially resistant microorganisms in intubated patients with hospital-acquired pneumonia: the interaction of ecology, shock and risk factors. Intensive Care Med. 39(4), 672-681 (2013).

68 Gastmeier P, Sohr D, Geffers C, Ruden H, Vonberg RP, Welte T. Early- and late-onset pneumonia: is this still a useful classification? Antimicrob. Agents Chemother. 53(7), 2714-2718 (2009)

69 Medicines and Healthcare Products Regulatory Agency. Public Assessment Report. Zevtera $500 \mathrm{mg}$ powder for concentrate for solution for infusion (UK/H/5304/001/DC) 2013. http://www.mhra.gov.uk

70 Frampton JE. Ceftaroline fosamil: a review of its use in the treatment of complicated skin and soft tissue infections and communityacquired pneumonia. Drugs 73(10), 1067-1094 (2013)

71 Kaye KS, Udeani G, Cole P, Friedland HD. Ceftaroline fosamil for the treatment of hospital-acquired pneumonia and ventilatorassociated pneumonia. Hosp. Pract. 1995, 1-6 (2015). 